\title{
Messung des Gesundheitszustands anhand Charts Lebensqualität von Patienten im ärztlichen Notfalldienst
}

\author{
H. Sandholzer, Th. Karg, Selbständige Abteilung für Allgemeinmedizin der Medizinischen Fakultät, Universität Leipzig
}

NOTFALLMEDIZIN 2003; 29: 246-254

Über die Lebensqualität von Patienten, die außerhalb der normalen Sprechstundenzeit den ärztlichen Notdienst aufsuchen, ist wenig bekannt. Ziel einer Untersuchung war die Ermittlung des funktionellen Gesundheitszustands in Abhängigkeit von der Inanspruchnahmezeit, der Beschwerden, Diagnosen und ärztlichen Leistungen, des Krankheitsschweregrads und der Dringlichkeit der Behandlung sowie des Verlaufs. Die gesundheitsbezogene Lebensqualität kann schnell, reliabel und gut mit den so genannten Wonca-Charts-plus-2 gemessen werden. Die hier gemessenen Werte liegen deutlich außerhalb des Bereichs, der von normalen Kontrollpersonen erreicht wird. Die Diskussion um eine inadäquate Inanspruchnahme des ärztlichen Notdienstes greift ohne Berücksichtigung der Lebensqualität und der Behandlungszufriedenheit der Patienten ins Leere.

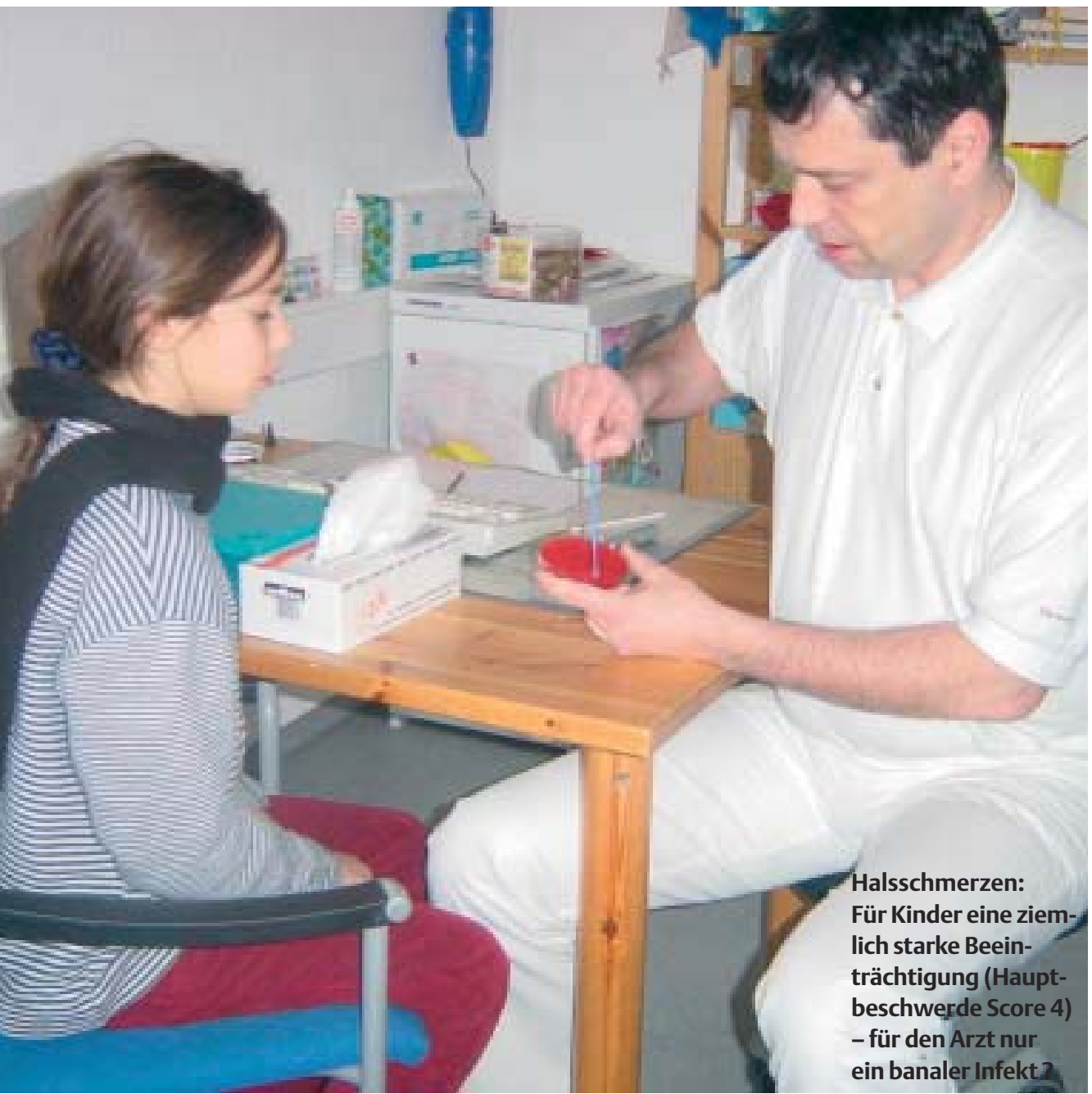

$\mathrm{D}$ er ärztliche Notdienst ist ein unverzichtbarer Bestandteil jedes Gesundheitssystems, der die medizinische Versorgung der Bevölkerung außerhalb der normalen Arbeitszeiten („Out of hours“) sicherstellt. Weltweit stellt sich den praktisch tätigen Ärzten vor Ort als auch der Gesundheitsadministration die Frage nach einer angemessenen Versorgung außerhalb der normalen Sprechstundenzeit. So liegt eine Fülle von Studien, Diskussionsbeiträgen und Büchern zum Thema vor (Carney 1999; Christensen and Olesen 1995; Hallam 1994; Salisbury 2000; Tönies 1991). Erstaunlicherweise widmen sich nur wenige Beiträge den subjektiven Aspekten, das heißt welche Gründe die Patienten haben, den Notdienst aufzusuchen, wie sie sich fühlen, ob sie mit der Versorgung zufrieden sind und ob sich ihr Zustand subjektiv bessert. In der vorliegenden Arbeit werden die Inanspruchnahmegründe und der funktionelle Zustand als Teil des übergeordneten Konzepts der gesundheitsbezogenen „Lebensqualität“" (Health Related Quality of Life) von Patienten beschrieben, die den ärztlichen Notdienst aufsuchen.

\section{Methodik}

Für die Einschätzung der gesundheitsbezogenen Lebensqualität stehen klinische Standardinstrumente zur Verfügung, zum Beispiel das Nottingham Health Profile (NHP), das Sickness Impact Profile (SIP), das Medical Outcomes Study Short-Form 36 Item Instrument (SF36), das EuroQoL, Dartmouth Primary Care Cooperative Information Project (COOP) chart system, der European Organization for Research and Treatment of Cancer (EORTC) 


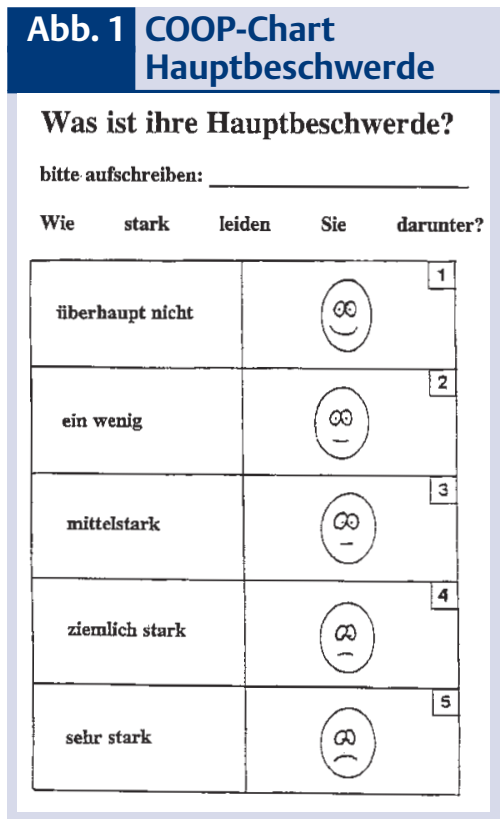

Quality of Life Questionnaire (QLQ) und das WHOQOL (Anderson et al. 1996). Ausgehend von der WHODefinition der Gesundheit empfiehlt die Weltorganisation der Allgemeinärzte die „WONCA COOP CHARTS“ zum Erfassen der psychischen, physischen und sozialen Gesundheit (Nelson et al. 1987; Nelson et al. 1990; Scholten and van Weel 1992). Es handelt sich dabei um ein sehr anschauliches Instrument, das Piktogramme, verbale Beschreibungen, Schweregradscores und Eigenschaften von visuellen Analogskalen verbindet (Abb. 1). Die Testgüte wurde sowohl international $(8,9)$ als auch bei deutschen Patienten (Sandholzer 1992) evaluiert, der klinische Nutzen in Querschnitts- (Nelson et al. 1987), Längsschnitts- (Nelson et al. 1990) und randomisierten (Wasson et al. 1992) Studien belegt. Beurteilt werden Auswirkungen von Gesundheitsstörungen auf den Gesundheitszustand, die Stimmung, die sozialen und alltäglichen Aktivitäten, die körperliche Leistungsfähigkeit und die Veränderung des Gesundheitszustands. Optionale Charts beziehen sich unter anderem auf den Beratungsanlass (Art und Schweregrad der Beeinträchtigung) und die empfundenen Schmerzen.

Es erwies sich als ungünstig, die Einschätzung der Gesundheit auf den Zeitraum der letzten zwei Wochen zu beziehen (Peters et al. 1998;

\section{Tab. 1 Patientengut}

\begin{tabular}{|c|c|c|}
\hline Variablen & $\begin{array}{l}\text { Häufigkeits- } \\
\text { verteilung (\%) }\end{array}$ & $\begin{array}{l}\text { Mittlerer } \\
\text { Lebens- } \\
\text { qualitätsscore }\end{array}$ \\
\hline Geschlecht & & $\mathrm{P}=0,38$ \\
\hline Weiblich & 56,6 & 3,7 \\
\hline Männlich & 43,4 & 3,6 \\
\hline Alter & & $P=0,07$ \\
\hline $0-4$ & 10,9 & 3,7 \\
\hline $5-14$ & 6,6 & 3,2 \\
\hline $15-24$ & 10,9 & 3,5 \\
\hline $25-44$ & 30,1 & 3,6 \\
\hline $45-64$ & 22,7 & 3,7 \\
\hline $65-74$ & 8,6 & 3,7 \\
\hline$\geq 75$ & 10,2 & 4,0 \\
\hline Dringlichkeit & & $P=0,001$ \\
\hline Keine & 8,2 & 3,2 \\
\hline Ein Tag aufschiebbar & 21,1 & 3,4 \\
\hline Nicht aufschiebbar & 70,7 & 3,8 \\
\hline Schweregrad der Krankheit & & $P=0,000$ \\
\hline nicht/leicht beeinträchtigt & 7,4 & 3,2 \\
\hline Mäßig & 23,0 & 3,4 \\
\hline Schwer & 51,6 & 3,7 \\
\hline Sehr schwer & 18,0 & 4,1 \\
\hline Lebensbedrohlich & 0 & - \\
\hline Ort der Behandlung & & $P=0,000$ \\
\hline Praxis & 52,4 & 3,4 \\
\hline Hausbesuch & 47,6 & 3,8 \\
\hline Untersuchung & & $P=0,000$ \\
\hline Keine & 3,9 & 2,9 \\
\hline Gezielt & 40,8 & 3,4 \\
\hline Organuntersuchung & 34,5 & 3,8 \\
\hline Ganzkörperstatus & 20,8 & 4,0 \\
\hline Behandlung & & $P=0,084$ \\
\hline Keine & 8,6 & 3,8 \\
\hline Beratung & 18,0 & 3,5 \\
\hline Rezept & 32,4 & 3,6 \\
\hline Spritze & 31,3 & 3,8 \\
\hline Infusion & 2,3 & 4,2 \\
\hline Wundversorgung & 6,6 & 3,5 \\
\hline Notarztwageneinsatz & 0,8 & 3,1 \\
\hline Krankenhaus & & $P=0,000$ \\
\hline Ambulant & 87,9 & 3,4 \\
\hline Stationär & 12,1 & 4,1 \\
\hline
\end{tabular}

Sandholzer 1992). Wenn jemand montags akut krank ist und mittwochs wiederbestellt wird, kann sich an den COOP-Scores per definitionem nichts geändert haben. Diese „Unsensitivität“ zeigt sich beispielsweise bei Patienten mit akuter Lumboischialgie, die sich in den letzten Wochen glänzend gefühlt hatten, sich aktuell jedoch kaum rühren können und deswegen den Notdienst verständigen. Aus diesem Grund wurde das Zwei-Wochen-
Kriterium fallen gelassen und die Charts in der Version einer früheren Studie angewandt (Sandholzer 1992). Die Beeinträchtigung der Lebensqualität insgesamt wurde durch Mittelwertbildung der acht Items „Beeinträchtigung durch Hauptbeschwerde“, „Körperliche Leistungsfähigkeit“, „Stimmung“, „tägliche Aufgaben“, „Schmerzen“, „Kontakte zu Mitmenschen“, „Gesundheitszustand“ und „Veränderung des Gesundheitszustands“ ermittelt. 


\section{Abb. 2 Hauptbeschwerden nach Organsystem}

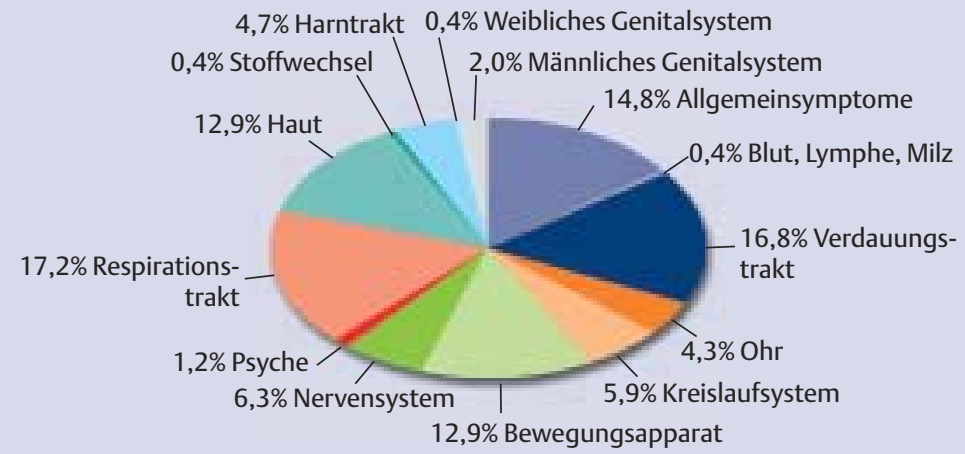

Einschätzung des Schweregrads der Hauptbeschwerde durch die Patienten

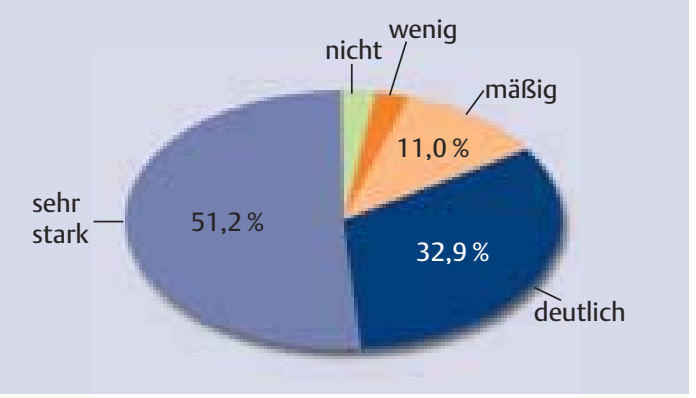

Die Patienten bekamen die Charts vor den Konsultationen in der Notfallpraxis von der Sprechstundenhilfe, beziehungsweise beim Hausbesuch durch den Arzt ausgehändigt. Erfasst wurden elf Dienste mit insgesamt 256 Konsultationen. Nach der Konsultation verteilte der Arzt einen zweiten Bogen mit demselben Instrument, Fragen zur Zufriedenheit mit der hausärztlichen und der notärztlichen Versorgung. Die Follow-up-Befragung war innerhalb einer Woche anonym auszufüllen und über einen beiliegenden Freiumschlag an den diensthabenden Arzt zu senden. Über eine Codenummer konnten die beiden Fragebögen zugeordnet werden. Der Arzt füllte seinerseits einen Fragebogen aus, der Daten aus den notwendigen Angaben des Abrechnungsformulars extrahierte (Alter, Geschlecht, Rufzeit, Diagnose, Hausbesuch). Ferner wurde die Beratungsursache des Patienten möglichst wortgetreu wiedergeben, diagnostische und therapeutische
Maßnahmen notiert. Ferner schätzen die Ärzte die Dringlichkeit der Behandlung sowie den Schweregrad des Gesundheitsproblems ein (Tab. 1). Beratungsursachen und Diagnosen wurden nach der ICPC verschlüsselt. Zur statistischen Auswertung wurde das Programm SPSSData-Entry und SPSS-PC (u.a. oneway, reliability) verwandt.

\section{Ergebnisse}

In zehn Diensten wurden insgesamt 256 Patienten aller Altersgruppen behandelt, wobei 203 Patienten den Fragebogen vollständig ausfüllten (Response quote 79\%). Bei über der Hälfte aller Patienten handelte es sich um Frauen. Bei 71\% der Patienten war der Dienst tuende Arzt der Meinung, dass es sich um eine dringliche Behandlung handelt, die nicht aufschiebbar ist, während bei 8\% eine Notwendigkeit zum Aufsuchen des Notdienstes nach ärztlicher Meinung nicht bestand, da ebenso gut der Hausarzt am darauffolgenden Werktag die Behandlung hätte übernehmen können (Tab. 1). Behandelt wurden Patienten mit allen Ausprägungsgraden von Gesundheitsstörungen mit Ausnahme von lebensbedrohlichen, reanimationspflichtigen Krankheiten. 12\% der Patienten wurden ins Krankenhaus eingewiesen beziehungsweise dort hinbegleitet.

Die erste Forschungsfrage betraf den Grund der Inanspruchnahme sowie eine Quantifizierung seines Schweregrads. Die Beratungsursachen betrafen alle Organsysteme (ICPC- Kapitel, siehe Abb. 2) und umfassten mehrheitlich Befindlichkeitsstörungen der Atemwege und des Gastrointestinaltrakts, Allgemeinsymptome wie zum Beispiel Fieber, Schmerzen bei Erkrankungen des Bewegungsapparats sowie Symptome der Haut wie beispielsweise Juckreiz, Verletzungen bei kleineren Unfällen. Die Rangliste nach Häufigkeit (Tab. 2) führten Fieber, Halsschmerzen, Koliken, Atemnot, Husten und Brennen beim Wasserlassen an. Dementsprechend wurden auch sehr häufig am Ende der Beratung Infektionen diagnostiziert.

Auf die Frage „Wie stark fühlen Sie sich dadurch (Hauptbeschwerde) beeinträchtigt?" gaben die Hälfte aller Patienten eine sehr starke und ein weiteres Drittel eine deutliche Beeinträchtigung durch ihre so genannten „banalen“ Beschwerden an (Abb. 3).

Welcher Beratungsanlass geht aus Sicht der Patienten mit großer, welcher mit eher geringer Einbuße im Bezug der Wonca-COOP-Charts einher? Zu diesem Zweck wurde ein Index aus den verwendeten acht COOP-Charts - unter Einschluss der optionalen Charts - gebildet. Eine Reliabilitätsanalyse zeigte, dass das Aufsummieren der acht Items statthaft ist (Cronbachs alpha $=0,82$, Splitt-half-Methode nach Guttman 0,85 ).

Die Lebensqualität variierte mit der Uhrzeit dergestalt, dass in den frühen Morgenstunden (4 bis $6 \mathrm{Uhr}$ ) die Patienten mit den größten Beeinträchtigungen kamen. Eine nähere Prüfung ergab, dass sich hier nicht nur ernstere Krankheiten wie Myokardinfarkt und Asthmaanfall, sondern auch Beschwerden mit hohem Leidensdruck fanden. Es ist daher spannend zu sehen, welche Beschwerden mit hoher Beeinträchtigung einhergehen.

Eine überdurchschnittlich starke Beeinträchtigung wiesen Patienten bzw. Patientinnen mit Beschwerden des weiblichen Genitaltrakts, der Psyche, des Verdauungsapparats, des Harntrakts und des Bewegungsapparats auf. In Tabelle 3 ist die „Hitliste“ der Beratungsanlässe im Bezug auf subjektive Beeinträchtigung der Lebensqualität dargestellt. Koliken, Herzenge und Schmerzen in der 
Brust, die vermutlich auf den Bewegungsapparat zurückgehen, gehören zu den stärksten Beeinträchtigungen (Werte 4 und mehr). Bauchschmerzen, Herzschmerzen und Fieber liegen mit Werten über $3 \mathrm{im}$ Bereich mittlerer Beeinträchtigung. Den Prototyp eher leichterer Beeinträchtigungen stellen Schnupfen oder Schnittwunden dar.

In Bezug auf die Lebensqualitätseinbuße führten Diagnosen von Erkrankungen der Psyche (Mittelwert $4,4 \pm 0,4 ; n=8)$, des Harntrakts $(4,1 \pm$ $0,6 ; \mathrm{n}=17)$ bzw. des Verdauungstrakts $(4,0 \pm 0,6 ; n=28)$ vor Erkrankungen des Bewegungsapparats (3,8 $\pm 0,6 ; n=21$ ), des Respirationstrakts $(3,6 \pm 0,6 ; n=50)$, des Kreislaufs (3,6 $\pm 0,7 ; n=16)$, Allgemeinerkrankungen $(3,5 \pm 0,7 ; n=27)$, des Nervensystems $(3,5 \pm 0,7 ; n=12)$, des Ohrs $(3,5 \pm 0,8 ; n=7)$ und der Haut (3,0 \pm 0,$8 ; \mathrm{n}=14$ ).

Kleinkinder und Höchstaltrige wiesen die deutlichsten Beeinträchtigungen auf (Tab. 1). Der sonst anzutreffende Geschlechterunterschied in der Selbsteinstufung der gesundheitsbezogenen Lebensqualität war im ärztlichen Notdienst nicht bedeutsam. Obwohl eine signifikante Beziehung zwischen der ärztlichen Einschätzung und der Selbsteinschätzung der Patienten bestand, war dieser Zusammenhang nicht allzu stark (Abb. 4): Der Arzt trifft auf Patienten, die er für sehr schwer krank und potentiell bedroht hält (Score 4 der Einschätzung), die sich selbst erstaunlich wenig beeinträchtigt fühlen (vier Patienten mit einen Score von weniger als 3); auch das Gegenteil kommt vor (Abb. 5). In Bezug auf die Dringlichkeit der Behandlung, Hausbesuchstätigkeit und den Aufwand der ärztlichen Untersuchung bestanden ebenfalls eindeutige Korrelationen mit dem Patientenzustand. Es bestand ein Trend zu stärkerer Beeinträchtigung mit dem Applikationsweg der medikamentösen Behandlung (Rezept, Spritze, Infusion). Die Ergebnisse der Nachbefragung zeigten, dass es den Patienten besser ging und die meisten mit der Behandlung zufrieden waren. Die Beeinträchtigung des funktionellen Zustands sank von einem mittleren Score von 3,7 auf
2,8, was statistisch signifikant war (t-test, $\mathrm{p}=0,001$ ).

\section{Diskussion}

Die Versorgung von Patienten außerhalb der normalen Sprechstundenzeit ist thematisch ein Dauerbrenner. Gesucht wird weltweit ein System, mit dem die Sicherstellung der ärztlichen Versorgung gewährleistet wird, wobei dies durch rotierende Dienste der Hausärzte, Ärztenetze, gemeinschaftliche Not- dienstpraxen, Notfallspezialambulanzen und Krankenhausambulanzen geregelt werden kann. Grundsätzlich bestehen mehrere Sichtweisen der Problematik. Kernpunkt der Diskussionen in verschiedenen Ländern ist es, eine Balance zwischen Zufriedenheit der Ärzte (Belastung durch den Dienst) und der Patienten (Erreichbarkeit und Bequemlichkeit, subjektive Beeinträchtigung, Zufriedenheit mit den Diensten) zu finden. Seit in Großbritannien und in Däne-

\section{Tab. 2 Rangliste der Beratungsursachen nach Häufigkeit}

\begin{tabular}{|c|c|c|c|}
\hline ICPC-Code Beratungsursache & $\mathbf{n}$ & $\%$ & $\begin{array}{l}\text { Kumu- } \\
\text { lierte } \\
\text { Prozente }\end{array}$ \\
\hline A03 Fieber & 21 & 8,2 & 8,2 \\
\hline R21 Beschwerden Rachen & 18 & 7,0 & 15,2 \\
\hline D01 Koliken & 13 & 5,1 & 20,3 \\
\hline R02 Dyspnoe & 12 & 4,7 & 25,0 \\
\hline R05 Husten & 11 & 4,3 & 29,3 \\
\hline U01 schmerzhafte Miktion & 10 & 3,9 & 33,2 \\
\hline H01 Ohrenschmerzen & 9 & 3,5 & 36,7 \\
\hline L02 Rückenschmerzen & 9 & 3,5 & 40,2 \\
\hline D02 Bauchschmerzen & 8 & 3,1 & 43,4 \\
\hline D10 Erbrechen & 8 & 3,1 & 46,5 \\
\hline N01 Kopfschmerz & 8 & 3,1 & 49,6 \\
\hline S02 Juckreiz & 8 & 3,1 & 52,7 \\
\hline A80 Unfälle & 7 & 2,7 & 55,5 \\
\hline K01 Herzschmerzen & 6 & 2,3 & 57,8 \\
\hline N17 Schwindel & 6 & 2,3 & 60,2 \\
\hline S07 generalisierte Rötung, Erythem der Haut & 6 & 2,3 & 62,5 \\
\hline A01 Schmerzen & 5 & 2,0 & 64,5 \\
\hline D11 Durchfall & 5 & 2,0 & 66,4 \\
\hline L04 Brustschmerz-Bewegungsapparat & 5 & 2,0 & 68,4 \\
\hline L08 Schulterbeschwerden & 5 & 2,0 & 70,3 \\
\hline D21 Schluckstörungen & 4 & 1,6 & 71,9 \\
\hline L03 Lumbago ohne Ausstrahlung & 4 & 1,6 & 73,4 \\
\hline L20 unspezifische Beschwerden mehrerer Gelenke & 4 & 1,6 & 75,0 \\
\hline S10 Karbunkel/Furunkel & 4 & 1,6 & 76,6 \\
\hline Y10 Unfruchtbarkeit & 4 & 1,6 & 78,1 \\
\hline D08 Blähungen & 3 & 1,2 & 79,3 \\
\hline K29 andere Brustschmerzen & 3 & 1,2 & 80,5 \\
\hline L17 Fußbeschwerden & 3 & 1,2 & 81,6 \\
\hline S18 Schnittverletzung & 3 & 1,2 & 82,8 \\
\hline A06 Synkope & 2 & 0,8 & 83,6 \\
\hline H03 Tinnitus & 2 & 0,8 & 84,4 \\
\hline K02 Herzenge & 2 & 0,8 & 85,2 \\
\hline K95 Varizen & 2 & 0,8 & 85,9 \\
\hline P28 geistige Behinderung & 2 & 0,8 & 86,7 \\
\hline S05 generalisierte Schwellung/Papel & 2 & 0,8 & 87,5 \\
\hline S17 Hautschürfung & 2 & 0,8 & 88,3 \\
\hline S19 andere Hautverletzungen & 2 & 0,8 & 89,1 \\
\hline S97 Ulcus cruris & 2 & 0,8 & 89,8 \\
\hline U07 andere Miktionsbeschwerden & 2 & 0,8 & 90,6 \\
\hline Rest & 24 & 9,0 & 100 \\
\hline
\end{tabular}


mark der Notdienst Teil von Reformen war, wissen wir nur wenig mehr über die Patientenzufriedenheit, die beispielsweise in Dänemark infolge der Reformen des Notdienstes sank (Olesen and Jolleys 1994).

Durch eine systematische Literaturrecherche unter Verwendung der Suchbegriffe „out of hours“ und ver- wandter Begriffe („emergency visit night house calls“) verknüpft mit „Quality of life“ Functional status wurden nur zwei Arbeiten zum Thema gefunden, wobei einmal das EuroQoL (Kersnik 2000) und einmal der SF36 (McKinley et al. 1997) eingesetzt wurde. Keine der beiden Studien beschäftigt sich mit dem

\section{Abb. 4 Krankheitsschweregrad nach Arzteinschätzung sowie Lebensqualität nach Patienteneinschätzung (mit Regressionslinie)}

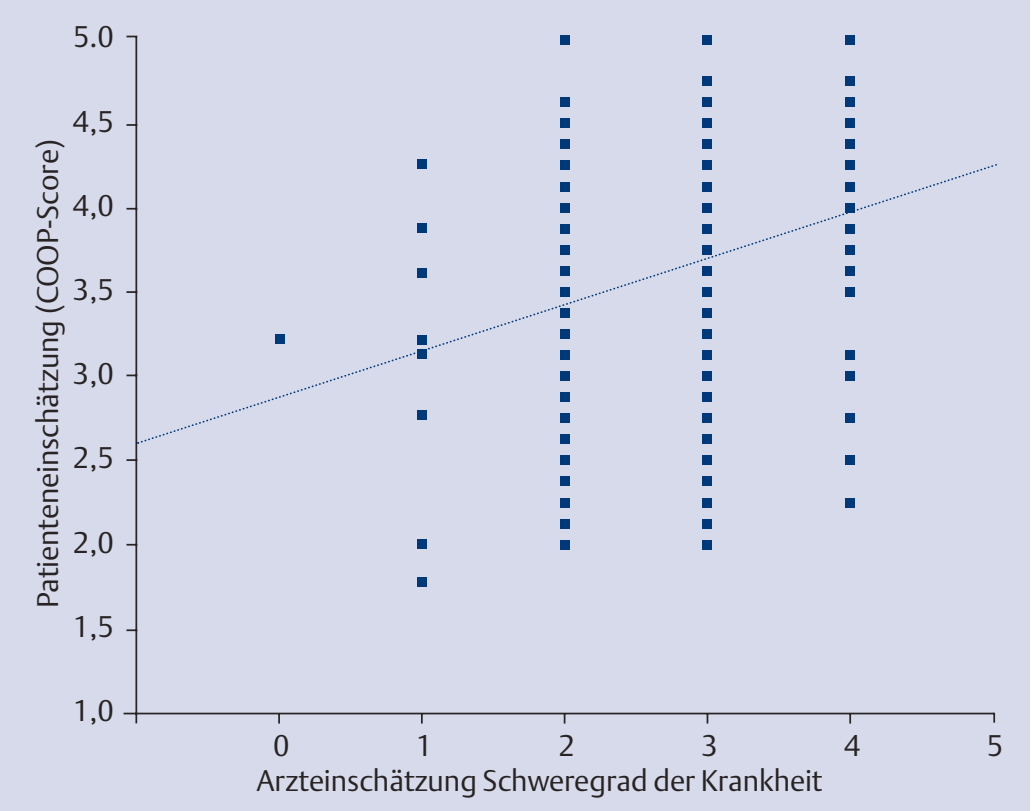

\section{Abb. 5 Variation der Lebensqualitäts-Scores mit der Uhrzeit} der Inanspruchnahme

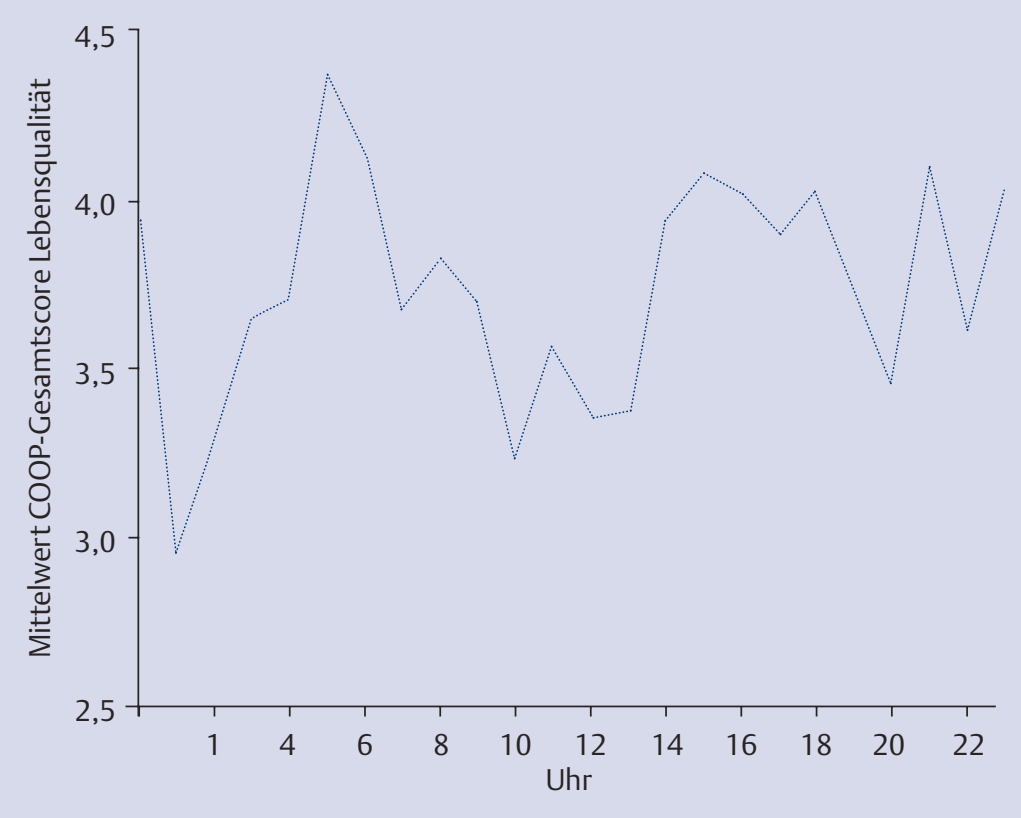

Gesundheitszustand aus der Sicht des Patienten zum Zeitpunkt der Inanspruchnahme, das heißt die befragten Patienten beurteilten ihren Zustand 24 bis 120 Stunden danach (McKinley et al. 1997) oder die Angabe des augenblicklichen Gesundheitszustands erfolgte in Bezug auf die Inanspruchnahme während eines ganzen Jahres (Kersnik 2000). Dies ist die erste Arbeit, die sich mit diesem Thema explizit beschäftigt und den funktionellen Zustand der Patienten aktuell zum Zeitpunkt der Inanspruchnahme maß.

Es ließ sich feststellen, dass die angegebenen Inanspruchnahmegründe prima vista vom banalen bis zum akuten neurologischen Defizit reichen. Quantitativ führen überwiegend harmlose Beschwerden an, wobei $84 \%$ der Patienten sehr stark beziehungsweise stark darunter litten. Die Beeinträchtigung des funktionellen Zustands wurde durch die Bildung eines Gesamtscores ermittelt, in den der Gesundheitszustand, die Stimmung (Angst, Depression), die sozialen Kontakte, die alltäglichen Aktivitäten (Beruf, Haushalt), die körperliche Leistungsfähigkeit, die Veränderung des Gesundheitszustands, der Schweregrad der Hauptbeschwerde und die empfundenen Schmerzen eingingen.

Bei Inanspruchnahme lag der Wert bei 3,7 und damit nahe im Bereich schwerer subjektiver Beeinträchtigung, besserte sich jedoch innerhalb einer Woche signifikant und näherte sich den Referenzwerten für Patienten, die während der normalen Sprechstundenzeit den Arzt aufsuchen, nicht jedoch von Kontrollprobanden ohne Inanspruchnahme einer Arztpraxis (Abb. 6). Aus diesem lässt sich ersehen, dass die Beeinträchtigung schwer war und somit die Patienten Grund hatten, den Notdienst zu rufen.

Es ließ sich feststellen, dass mit diesem Instrument der funktionelle Gesundheitszustand nicht nur bequem, schnell und mit großer Akzeptanz der Patienten erhoben werden konnte, sondern diese Einschätzung eine hohe interne Konsistenz aufwies und valide war. Der Einbezug der Items „Schweregrad der Hauptbeschwerden“, „Verände- 
rung des Gesundheitszustands“, „Stimmung“ und „Schmerzen“ ist für die Beurteilung von akut Kranken besonders wichtig.

Mit dieser Studie wurden erste Ergebnisse zur Auswirkung von Beschwerden und Krankheiten auf den funktionellen Gesundheitszustand im Notdienst erhoben. Für die Verbesserung der (Telefon-)Anamnese kann es wichtig sein, vorgebrachte Beschwerden nicht nur im Hinblick auf die potentielle Lebensgefährdung, sondern auch auf den subjektiven Leidendruck besser beurteilen zu können. Da die Beratungsanlässe und Diagnosen breit streuten, sind weitere Untersuchungen mit großen Fallzahlen notwendig.

Die Charts liegen in mehreren Sprachen vor und sind universell von medizinischen Profis einsetzbar. Da nun Normwerte vorliegen, sind weitere Einsatzgebiete denkbar:

- Als Screening und Triageinstrument

- Für eine rationellere und genauere Anamneseerhebung bei Patienten mit Kommunikationsproblemen

- Zur Verlaufsbeurteilung

- Um ein besseres Verständnis über die Determinanten des ärztlichen Notdienstes beizutragen, insbesondere zu erkennen, welche Rolle psychosoziale Faktoren wie Angst und Depressionen haben (Martin et al. 2002)

- Für eine verbesserte Diagnostik (Murphy and Lam 2001) von funktionellen Beeinträchtigungen, insbesondere psychosozialen Einschränkungen (Doetch et al. 1994; Wasson et al. 1994) und Schmerzen (Wasson et al. 1992)

- Zur Evaluation und Qualitätssicherung in Arztnetzen, z.B. Vergleich verschiedener Versorgungskonzepte im Notdienst (Cragg et al. 1997; Shepperd et al. 1998).

\section{Summary}

Little is known about health related quality of life of patients seeking urgent medical care. The aim of this study was (1) to describe the episodes of care using the ICPC classification and (2) to relate these data to the

\section{Tab. 3 Rangliste der Beschwerden in Bezug auf subjektive Beeinträchtigung}

\section{ICPC-Code Beratungsanlass}

Mittelwert

X08 Zwischenblutung

4,7500

N18 paralytische Schwäche

4,5059

U07 Miktionsbeschwerden

4,5059

P02 akuter Stresszustand

4,5000

D11 Durchfall

4,3482

L03 Lumbago ohne Ausstrahlung

4,2976

L20 unspezifische Beschwerden mehrerer Gelenke 4,2520

D01 Koliken

4,2436

N06 Missempfindungen Bewegungsstörungen $\quad 4,1309$

P28 geistige Behinderung $\quad 4,1309$

R05 Husten $\quad 4,1302$

L04 Brustschmerz-Bewegungsapparat $\quad 4,1000$

K02 Herzenge $\quad 4,0625$

R03 Keuchen $\quad 4,0059$

S97 Ulcus cruris $\quad 4,0059$

D06 lokaler Abdominalschmerz $\quad 4,0000$

D08 Blähungen $\quad 3,9404$

K86 Hypertonie $\quad 3,8809$

L15 Kniebeschwerden $\quad 3,8809$

D10 Erbrechen $\quad 3,8135$

L02 Rückenschmerzen $\quad 3,8078$

K01 Herzschmerzen 3,7956

D02 Bauchschmerzen $\quad 3,7864$

A80 Unfälle $\quad 3,7767$

U01 schmerzhafte Miktion $\quad 3,7552$

L17 Fußbeschwerden $\quad 3,7539$

N01 Kopfschmerz $\quad 3,7529$

A03 Fieber $\quad 3,6512$

A01 Schmerzen allgemein $\quad 3,6309$

L08 Schulterbeschwerden $\quad 3,5833$

H03 Tinnitus $\quad 3,5059$

S17 Hautschürfung $\quad 3,5059$

R02 Dyspnoe 3,4594

R21 Beschwerden Rachen $\quad 3,4563$

A06 Synkope $\quad 3,4434$

D21 Schluckstörungen $\quad 3,4375$

H01 Ohrenschmerzen $\quad 3,4226$

L01 Nackenschmerzen $\quad 3,3750$

K29 andere Brustschmerzen $\quad 3,2559$

S09 Paronychie $\quad 3,2559$

S05 generalisierte Schwellung Papel $\quad 3,1934$

R97 Heuschnupfen 3,1309

S18 Schnittverletzung $\quad 3,1309$

L05 Flankenbeschwerden $\quad 3,1250$

N17 Schwindel $\quad 3,0684$

S07 generalisierte Rötung, Erythem der Haut $\quad 3,0059$

S02 Juckreiz 2,9583

S19 andere Hautverletzungen $\quad 2,8809$

S10 Karbunkel/Furunkel $\quad 2,8392$

A08 unspezifische Schwellung $\quad 2,7559$

R07 Schnupfen $\quad 2,5000$

A04 Schwächegefühl $\quad 2,3750$

Y10 Unfruchtbarkeit $\quad 2,1309$

K25 Angst vor Bluthochdruck 2,0059

S06 lokale Rötung/Erythem der Haut 2,0059 


Abb. 6 Lebensqualität von Notdienstpatienten im Vergleich
zu Referenzstichproben

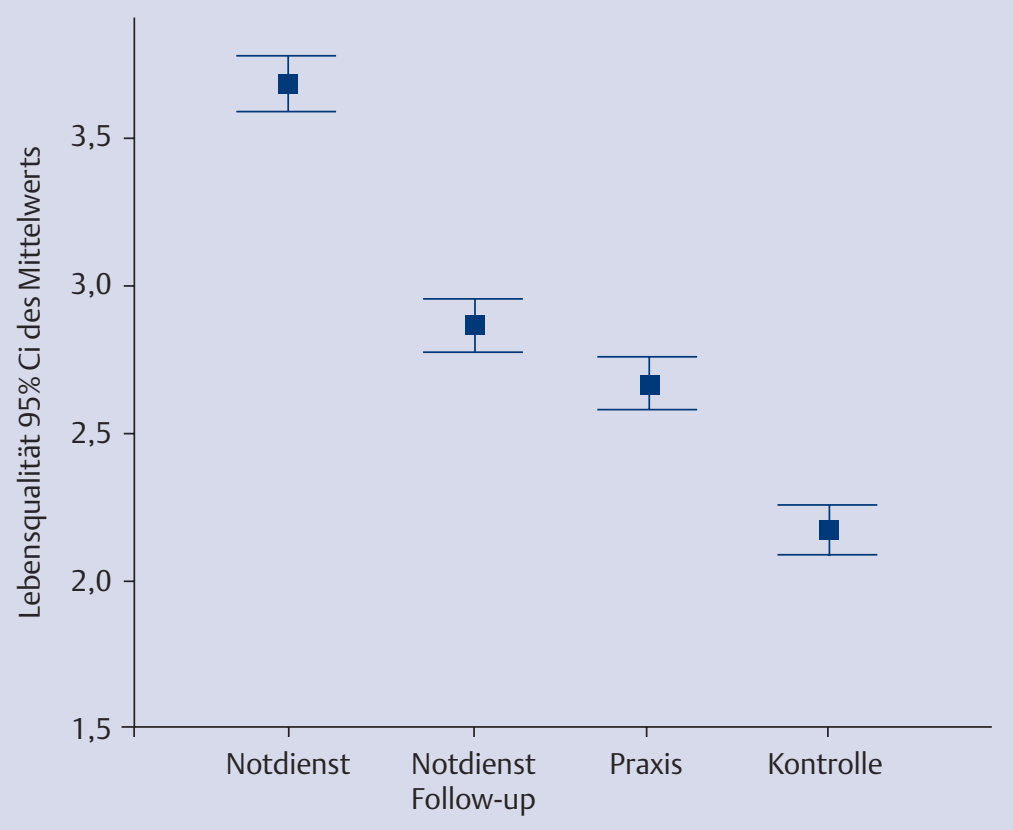

functional status measured by the 6 Wonca-COOP-Charts and two optional charts for main and the main complaint chart. 256 patients seeking medical care to a community based emergency service during 11 weekends. The most common reasons for encounter were fever, sore throat, abdominal pain and shortness of breath. The functional status of these patients was moderately to severely impaired and related both to the severity and urgency of the health problem and to the process of care (need for thorough examination by the physician, home visits, hospital admission). In conclusion the study supports that the usage of the WoncaCOOP-Charts in the assessment of quality of emergency care. The discussion about appropriateness of emergency services - which hitherto is based only on doctors appraisal of health status - needs supplementation with the patients point of view.

\footnotetext{
Literatur

1. Anderson RT, Aaronson NK, Bullinger $M$, McBee WL. A review of the progress towards developing health-related qualityof-life instruments for international clinical studies and outcomes research. Pharmacoeconomics1996; 10: 336-355

2. Carney CJ. Prehospital care - A UK perspective. Br.Med.Bull 1999; 55: 757-766
}

3. Christensen MB, Olesen F. On-duty medical service regulation in Norway, Sweden, Finland and Denmark. Nord.Med. 1995; 110: 127-131

4. Cragg DK, McKinley RK, Roland MO et al. Comparison of out of hours care provided by patients' own general practitioners and commercial deputising services: a randomised controlled trial. In: The process of care. BMJ 1997; 314: 187-189

5. Doetch TM, Alger BH, Glasser M, Levenstein J. Detecting depression in elderly outpatients: findings from depression symptom scales and the Dartmouth COOP charts. Fam.Med. 1994; 26: 519-523

6. Hallam L. Primary medical care outside normal working hours: review of published work. BM] 1994; 308: 249-253

7. Kersnik J. Observational study of home visits in Slovene general practice: patient characteristics, practice characteristics and health care utilization. Fam.Pract. 2000; 17: 389-393

8. Kinnersley P, Peters T, Stott N. Measuring functional health status in primary care using the COOP-WONCA charts: acceptability, range of scores, construct validity, reliability and sensitivity to change. Br.J.Gen. Pract. 1994; 44: 545-549

9. Lindegaard PM, Bentzen N, Christiansen T. Reliability of the COOP/WONCA charts. Test-retest completed by patients presenting psychosocial health problems to their general practitioner. Scand.J.Prim. Health Care 1999; 17: 145-148

10. Martin A, Martin C, Martin PB, Martin PA et al. 'Inappropriate' attendance at an accident and emergency department by adults registered in local general practices: how is it related to their use of primary care? J.Health Serv.Res.Policy 2002; 7: 160-165
11. McKinley RK, Cragg DK, Hastings AM et al. Comparison of out of hours care provided by patients' own general practitioners and commercial deputising services: a randomised controlled trial. II: The outcome of care. BMJ 1997; 314: 190-193

12. Murphy DD, Lam CL. Feasibility and acceptability of the COOP/WONCA charts for identification of functional limitations in rural patients of the People's Republic of China. Int.J.Rehabil.Res. 2001; 24: 207-219 13. Nelson E, Wasson J, Kirk J et al. Assessment of function in routine clinical practice: description of the COOP Chart method and preliminary findings. J.Chronic.Dis. 1987; 40 Suppl 1: 55-69

14. Nelson EC, Landgraf JM, Hays RD et al. (1990). The functional status of patients. How can it be measured in physicians' offices? Med.Care 1990; 28: 1111-1126

15. Olesen F, Jolleys JV. Out of hours service: the Danish solution examined. BMJ 1994; 309: 1624-1626

16. Peters TJ, Coast J, Richards SH, Gunnell D]. Effect of varying the time frame for COOP-WONCA functional health status charts: a nested randomised controlled trial in Bristol, UK. J.Epidemiol.Community Health 1998; 52: 59-64

17. Salisbury C. The demand for out-ofhours care from GPs: a review. Fam.Pract. 2000; 17: 340-347

18. Sandholzer H. Die Beurteilung der Lebensqualität von Allgemeinpraxispatienten. Z Allg Med 1992; 68: 335-341

19. Scholten JHG, van Weel C. Functional status assessment. Den Haag: WONCA, 1992

20. Shepperd S, Harwood D, Jenkinson C et al. Randomised controlled trial comparing hospital at home care with inpatient hospital care. I: three month follow up of health outcomes. BMJ 1998; 316: 17861791

21. Tönies H. Hausbesuch und Diagnostik im Notdienst. Berlin, Heidelberg, New York: Springer, 1991

22. Wasson J, Hays R, Rubenstein, L et al. The short-term effect of patient health status assessment in a health maintenance organization. Qual.Life Res. 1992; 1: 99-106 23. Wasson JH, Kairys SW, Nelson EC et al. A short survey for assessing health and social problems of adolescents. Dartmouth Primary Care Cooperative Information Project (The COOP). J.Fam.Pract. 1994; 38: 489494

\section{Anschrift des Verfassers}

Prof. Dr. med. habil. Hagen Sandholzer Leiter der selbständigen Abteilung für Allgemeinmedizin

Medizinische Fakultät

Phillip-Rosenthalstr. 27a

04103 Leipzig

Fax: 0341/9715-719

E-Mail: haeb.medizin.uni-leipzig.de 